\title{
VARIATION SPATIALE DU RISQUE POUR LES PORCS DE CONTRACTER LA TRYPANOSOMOSE DANS LA ZONE PÉRIURBAINE DE KINSHASA
}

\author{
SUMBU J.*, DE DEKEN R.**, DECKERS N.**, MPIANA S.*, KABAMBI P.*, TSHILENGE G.* \& BOELAERT M.**
}

Summary: SPATIAL VARIATION OF RISK FOR PIGS TO CONTRACT TRYPANOSOMOSIS IN FARMS SITUATED IN THE PERI-URBAN AREA OF KINSHASA

In an effort to understand better the transmission risk as well for the animal african trypanosomosis (AAT) as for the human trypanosomosis (HAT) in the peri-urban zone of Kinshasa, a serologic study was carried out in local pig farms from 2003 to 2005. An indirect ELISA was used to detect the presence of trypanosome antibodies in 1,240 pigs originating from

404 farms. Seropositivity was recorded in 155 farms (38 \%), but varied considerably according to the district. In $6 \%$ of the farms TAA could be confirmed by parasitological examination. Trapping sites ( $n=367$ ) established in the neighbourhood of pig farms made it possible to capture 1,935 tsetse flies /Glossina fuscipes quanzensis). Among 562 dissected flies 23 were found to harbour trypanosomes resulting in an infection rate of $4.1 \%$. In the majority of the districts the transmission risk for animal trypanosomosis anticipated from the apparent vector densities was corroborated by the serology. Zones with strong indications of local AAT transmission were identified in several quarters of three peri-urban districts of Kinshasa: Mount-Ngafula, Ngaliema and N'Sele. An intensification of tsetse control activities in those sites of increased transmission risk is essential.

KEY WORDS : animal african trypanosomosis, pig, glossina, Kinshasa, risk area, Democratic Republic of Congo, human african trypanosomosis.

I es trypanosomoses africaines sont un problème majeur de santé publique et animale en République Démocratique du Congo (RDC). La maladie du sommeil était connue comme n'affectant que les populations rurales. Toutefois, entre 1996 à 2000, 2451 nouveaux cas de trypanosomose humaine africaine (THA) parasitologiquement confirmés ont été déclarés dans la province "Ville de Kinshasa", 956 (39\%) des patients résidaient dans la zone urbaine (Ebeja et al., 2003). En 1999, une épidémie de THA a été signalée dans la commune de Kisenso en zone périurbaine (De Deken et al., 2005). Cette augmentation du nombre de cas chez des résidents de la ville de Kinshasa a posé la question de la localisation de la transmission dans l'espace rural, périurbain ou urbain.

\footnotetext{
* Laboratoire Vétérinaire de Kinshasa, Kinshasa, République Démocratique du Congo.

** Institut de Médecine Tropicale d'Anvers, Belgique. Correspondance: Redgi De Deken, Insitute of Tropical Medicine of Antwerp, Nationalestraat 155, 2000 Antwerp, Belgium.

Tél. : + 32 (0) 32476270 - Fax : 32 (0) 32476268.

E-mail : rddeken@itg.be
}

\section{Résumé :}

Afin de mieux comprendre le risque de transmission de la trypanosome animale africaine (TAA) et humaine (THA) en zone périurbaine de Kinshasa, une étude sérologique a été réalisée dans des élevages porcins entre 2003 et 2005. Un test ELISA indirect a été utilisé pour détecter la présence d'anticorps dirigés contre les trypanosomes sur un échantillon de 1240 porcs provenant de 404 fermes. 155 fermes (soit 38 \%) se sont révélées séropositives avec une grande variation suivant la commune. Dans $6 \%$ des fermes, des cas de TAA ont pu être démontrés par l'examen parasitologique. Des piégeages ( $n=367$ ) aux alentours des fermes ont permis de capturer 1935 mouches tsé-tsé (Glossina fuscipes quanzensis). Sur 562 mouches disséquées, 23 contenaient des trypanosomes soit un taux d'infestation de $4,1 \%$. Le risque de transmission de la trypanosomose animale, escompté à partir des captures du vecteur, est confirmé dans la plupart des communes par les résultats sérologiques. Des zones à forte probabilité de transmission de TAA ont été identifiées dans plusieurs quartiers situés dans trois communes périurbaines de Kinshasa: Mont-Ngafula, Ngaliema et N'Sele. Une intensification de la lutte anti-vectorielle s'impose dans les endroits à risque.

\begin{abstract}
MOTS CLÉS : trypanosomose animale africaine, porc, glossines, Kinshasa, zones à risque, République Démocratique du Congo, trypanosomose humaine africaine.
\end{abstract}

Les données entomologiques collectées à partir des années 1991 dans la ville de Kinshasa ont démontré que le vecteur, Glossina fuscipes quanzensis (Pires, 1948), se trouvait non seulement à la périphérie de la ville, mais également là où la rivière N’Djili traverse les zones urbaines (Ebeja et al., 2003). Une étude prospective plus récente a mis en évidence qu'autour de Kinshasa les glossines sont essentiellement trouvées le long de certaines grandes rivières traversant la zone rurale et périurbaine de Kinshasa, lesquelles leurs offrent un habitat favorable. La partie urbaine proprement dite est restée largement libre de glossine (De Deken et al., 2005).

D'après Robays et al. (2005), les cas de THA observés chez les résidents de la partie urbaine de Kinshasa entre 2004 et 2005 sont pour la plupart des cas importés, l'infection ayant été acquise dans une autre province ou dans la zone rurale de Kinshasa lors d'activités commerciales et agropastorales. Toutefois, il existe une forte présomption de transmission locale en zone périurbaine à Kisenso en 1999 (De Deken et al., 2005). 
La région administrative "Ville de Kinshasa" a un statut de province et comprend la zone urbaine proprement dite, une zone périurbaine assez densément peuplée, et une zone rurale peu peuplée qui est un foyer connu de THA. La population urbaine de Kinshasa dépend pour son approvisionnement en denrées alimentaires (telles légumes, viande, poissons,...) en grande partie de la production agropastorale et maraîchère de l'espace périurbain et du plateau des Bateke. L'élevage de porc domestique, très rentable, est très courant dans cette zone périphérique de Kinshasa.

Une étude menée au Cameroun démontre que le porc domestique est un réservoir potentiel de $T$. b. gambiense dans le foyer de THA de Fontem (Nkinin et al., 2002; Simo G. et al., 2006). En outre, le réservoir animal de la THA a été évoqué par plusieurs auteurs en République Démocratique du Congo (RDC) (Schares \& Mehlitz, 1996; Makumyaviri et al., 1989; Kageruka et al., 1977). Greggio, en 1917, avait déjà suggéré le rôle des porcs dans l'épidémiologie de la THA dans la vallée de l'Ikinsi, où la maladie du sommeil avait ravagé plus de la moitié de la population dans un environnement peu propice pour le vecteur mais où l'élevage porcin était bien implanté. Cependant, le rôle exact du porc comme réservoir de la THA est toujours controversé (Njiokou, 2006; Jammoneau et al., 2004 ). Si le porc jouait vraiment un rôle important dans le cycle de transmission de la THA, on s'attendrait à ce que les zones, où le risque de transmission pour les animaux est élevé, coïncident avec les zones où la THA se manifeste de façon sporadique. En ce qui concerne la province "Ville de Kinshasa", les données épidémiologiques sur les trypanosomoses porcines sont quasi inexistantes. La présente étude examine la distribution de la TAA dans les élevages porcins autour de Kinshasa en relation avec les données entomologiques afin de déterminer les zones à haut risque pour la transmission de la trypanosomose animale et de faciliter la recherche sur la transmission de la THA.

\section{MATÉRIELS ET MÉTHODES}

\section{AIRE D'ÉTUDE}

I a province "Ville de Kinshasa" est constituée de 24 communes à superficies très inégales (figure 1). L'étude s'est concentrée sur la zone périurbaine de Kinshasa qui fait la transition vers la partie rurale (figure 2).

\section{ANIMAUX}

L'étude s'est déroulée dans différents élevages et a porté exclusivement sur l'espèce porcine, type d'élevage de loin le plus important dans la zone. Il est à noter que $80 \%$ des élevages porcins sont implantés dans les zones périphériques et conduits d'une façon semi intensive avec des animaux issus de différents croisements, tenus et nourris dans des porcheries traditionnelles. La race Large White et ses croisements sont dominants. Le nombre d'animaux par exploitation varie entre cinq et 250 avec en moyenne 28 animaux par exploitation.

\section{IDENTIFICATION DES FERMES}

Les fermes ont été sélectionnées de façon aléatoire. L'adresse complète de l'éleveur, les coordonnées géographiques de la porcherie relevées avec un GPS (Garmin GPS 72), la distance de la ferme jusqu'au réseau hydrographique, l'état sanitaire, l'âge et le sexe de chaque animal prélevé ont été enregistrés.

\section{ÉCHANTILLONNAGE ET PRÉLÈVEMENT}

Par ferme, la taille de l'échantillon a été fixée à $10 \%$ de l'effectif total. Le sang a été prélevé au niveau de la veine auriculaire dans un tube avec anticoagulant (EDTA) pour l'examen parasitologique et sur papier filtre Whatman $n^{\circ}$ IV pour le test ELISA indirect.

Le sang prélevé a été gardé au froid. Au laboratoire, des microtubes hématocrites ont été remplis de ce sang et centrifugés. La recherche des trypanosomes au microscope a été fait suivant la méthode de Murray (Murray et al., 1977).

\section{Test sérologique}

Un test ELISA a été utilisé pour la détection d'anticorps contre les trypanosomes chez les 1421 porcs prélevés dans les différentes fermes. Le test a été réalisé à Kinshasa d'après un protocole d'ELISA indirect défini pour la détection des anticorps contre Trichinella spiralis du porc (Diouf, 2000), et adapté pour la détection des anticorps contre les trypanosomes en utilisant un antigène dérivé du stock CP 81 de T. congolense (Deckers, 2004). Les échantillons de sang ont été dupliqués et les sérums de contrôle quadruplés.

Les résultats sont exprimés en valeurs de pourcentage de positivité (PP). Le PP a été déterminé en divisant la densité optique moyenne de deux mesures successives de chaque échantillon par la médiane de densité optique d'un sérum de contrôle très positif (Wright et al., 1993). Le sérum de contrôle très positif a été obtenu à partir d'un porc Landrace Belge infecté expérimentalement avec T. congolense (Deckers, 2004) tandis que le sérum de contrôle négatif a été prélevé chez le même porc avant l'inoculation.

Le PP, au dessus duquel un porc a été considéré comme ayant été en contact avec des trypanosomes, a été calculé à partir de la densité optique de sérums de 152 porcs qui n'ont jamais été en contact avec des trypanosomes. 
La moyenne de PP des sérums négatifs plus trois écarttype a été retenue comme seuil de positivité, soit un seuil de PP égal ou supérieur à $32 \%$ avec une probabilité au dessus de $99 \%$. Si on prend comme seuil la moyenne de PP de sérums de porcs, qui n'ont jamais été en contact avec la maladie, plus deux écart-type, le seuil de PP diminue à $26 \%$ avec une probabilité au dessus de $95 \%$. Pour l'objectif de l'étude, qui était d'identifier les zones à haut risque de transmission, les faux positifs sont à éviter et seul les sera ayant un PP de $32 \%$ ou plus ont été considérés comme positifs. Cependant l'antigène préparé à partir du T. congolense ne réussit pas toujours à détecter des titres bas d'anticorps (Luckins 1977; Luckins \& Mehlitz, 1978). Pour cette raison, les sera ayant un PP entre 26 et $31 \%$ ont été considérés comme étant douteux tandis que ceux dont les résultats étaient inférieurs à $26 \%$, ont été considérés comme négatifs.

\section{REgROUPEMENT DE FERMES POSITIVES}

Le module "Risk adjusted nearest neighbour hierarchical spatial clustering" du programme Crimestat II version 2.0 (Levine, 2002) a été utilisé dans l'analyse d'agrégation (clustering) des cas. L'analyse recherche les fermes séropositives (PP moyens au dessus de $32 \%$ ) qui, en comparaison avec la distribution générale de toutes les fermes visitées, montrent une tendance à se regrouper. L'analyse donne donc des indications spatiales sur l'intensité de la transmission.

\section{CAPTURE DES GLOSSINES}

Cette étude s'est accompagnée d'un volet entomologique. Un piégeage systématique auprès des élevages visités a été réalisé. Les prospections se sont dérou- lées dans les bassins des rivières N'Djili, Lukunga, Lukaya et leurs affluents, et ceux des rivières Tshenke, Lutendele, Boye, Funa et Bumbu. Les critères de choix des sites de piégeage ont été déterminés en tenant compte de la proximité de l'élevage (moins de 100 mètres), de la présence de couvert végétal (galeries forestières) et le cas échéant de la présence de cours d'eau.

En moyenne, huit pièges Lancien et Goutteux du type BCT (Bureau Central de la Trypanosomiase) ont été posés dans chaque site. 46 sites situés dans neuf communes (Kimbanseke, Kisenso, Limete, Masina, MontNgafula, Ndjili, Ngaliema, Nsele, Selembao) ont été explorés. Au total 367 séances de piégeages ont été réalisées. Le piégeage s'est effectué pendant sept mois en 2003 (de juin à décembre) et pendant six mois en 2004 (de janvier à avril et de juillet à août). Il est à signaler que les pièges ont été posés pendant sept jours dans les zones à forte densité glossinienne et 14 jours dans les sites où la présence des mouches était très faible. La distance entre deux pièges a été de $50 \mathrm{~m}$. Le relevé des coordonnées géographiques des différents sites des piégeages a été assuré au moyen d'un GPS (Garmin GPS 72) et les glossines capturées vivantes ont été disséquées pour rechercher la présence des Trypanosoma spp.

\section{RÉSULTATS}

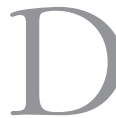
e 2003 à 2004, 418 fermes, soit environ $65 \%$ des élevages répertoriés dans la partie périurbaine de la "Province Ville de Kinshasa" et totalisant un effectif de 11234 porcins, ont été inclues dans l'étude.

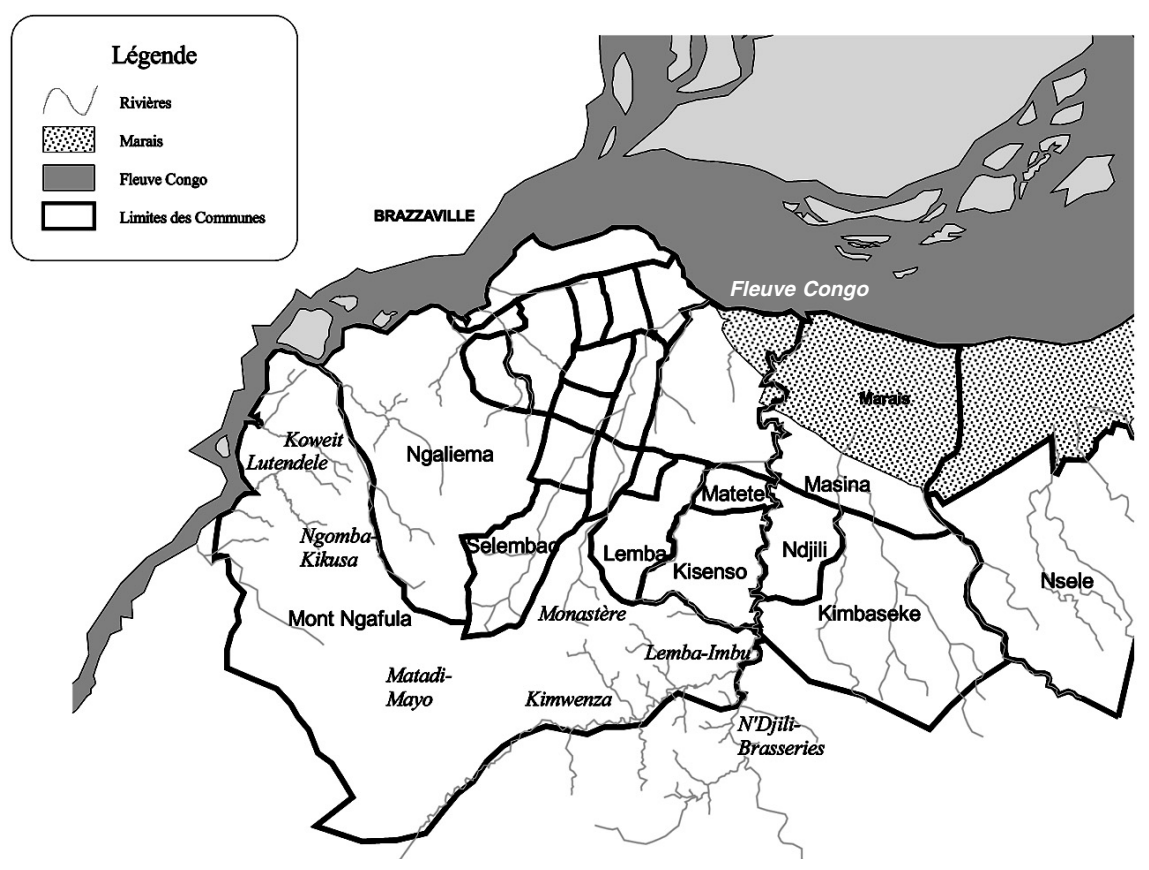

Fig. 1. - Communes et quartiers (en italique) de Kinshasa mentionnés dans l'étude. 


\begin{tabular}{lcrcc}
\hline Commune & $\begin{array}{c}\text { Nombre } \\
\text { de fermes } \\
\text { visitées }\end{array}$ & $\begin{array}{c}\text { Effectif } \\
\text { porcin }\end{array}$ & $\begin{array}{c}\text { Nombre } \\
\text { des fermes } \\
\text { positives }\end{array}$ & $\begin{array}{c}\text { \% des } \\
\text { fermes } \\
\text { positives }\end{array}$ \\
\hline Kimbaseke & 46 & 1023 & 0 & 0 \\
Kisenso & 29 & 547 & 0 & 0 \\
Limete & 16 & 315 & 0 & 0 \\
Masina & 17 & 182 & 0 & 0 \\
Matete & 6 & 27 & 0 & 0 \\
Mont-Ngafula & 165 & 5388 & 20 & 12 \\
N'Djili & 15 & 160 & 0 & 0 \\
Ngaliema & 54 & 1645 & 2 & 4 \\
N'Sele & 39 & 996 & 1 & 3 \\
Selembao & 31 & 971 & 0 & 0 \\
\hline Total & 418 & 11254 & 23 & 6 \\
\hline
\end{tabular}

Tableau I. - Résultats des tests parasitologiques réalisés dans les fermes des différentes communes.

\section{EXAMEN PARASITOLOGIQUE}

Le tableau I montre le nombre d'élevages porcins visités par commune et le pourcentage d'élevages dans lesquels l'examen parasitologique (Woo, 1970) a permis de détecter des porcs parasités par des trypanosomes. Sur les 418 fermes visitées, 23 (6\%) contenaient des porcs infestés et celles-ci se trouvaient essentiellement à Mont-Ngafula dans les quartiers Lutendele (rivières Lutendele et Nzimbi), Ngombe Lutendele (le long de la rivière Boyé) et Kimwenza (le long de la rivière Lukaya). Les deux fermes positives à Ngaliema se trouvaient le long de la rivière Lukunga et celle à N'Sele a été détectée dans le bassin de la rivière N'Djili.

\section{Test SÉROlOgique}

Le tableau II présente les résultats du test sérologique (ELISA indirect) les fermes étant classées comme positives, négatives et douteuses selon le pourcentage de positivité (PP). Les anticorps dirigés contre les trypa- nosomes ont été détectés chez des porcs élevés dans des communes où la présence de mouches tsé-tsé a été confirmée, mais aussi dans la commune de Selembao où $55 \%$ des fermes sont positives bien qu'aucune mouche n'y ait été capturée. En moyenne 38 \% des fermes prospectées sont affectées par le problème de TAA. Ramené à la population porcine $45 \%$ d'animaux prélevés se sont révélés sérologiquement positifs La figure 2 représente la distribution des fermes sérologiquement positives. La plupart se trouvent en zone périurbaine ou rurale, certaines dans l'espace urbain. Aucune influence de l'âge ou du sexe sur le résultat de la sérologie ne fut observée, mais il faut admettre que les porcelets ont été exclus de l'étude.

La figure 2 illustre également les résultats de l'étude d'agrégation. Les zones où au moins cinq fermes séropositives se regroupent de façon non-aléatoire sont représentées par un ovale. Ces zones constitueraient des sites où les porcs sont relativement plus exposés aux piqûres des glossines. Il s'agit des quartiers : Lutendele le long de la rivière Boyé et Lutendele, Ngomba Kikusa le long de la rivière Lukunga, Monastère le long de la rivière Funa, Kimwenza le long de la rivière Lukaya, Lemba Imbu en amont de la Lukaya et le long de la rivière N'Djili. Tous les cas de TAA parasitologiquement confirmés ont été dépistés dans ces mêmes endroits. La figure 3 indique les points où des glossines ont été capturées au cours de 13 mois de piégeage. Le nombre total de glossines capturées est de 1935, dont 1807 (93\%) proviennent des quartiers Kimwenza, Lemba Imbu, Matadi Mayo, Lutendele et Koweit de la commune de Mont-Ngafula. Les endroits où les captures ont été fructueuses se situent essentiellement le long des rivières où la galerie forestière est présente et forme un habitat adéquat pour l'espèce G. $f$. quanzensis. Parmi les 562 mouches disséquées, l'examen microscopique a permis de détecter 23 mouches contenant des trypanosomes, soit 4,1\%. Les études sur l'iden-

\begin{tabular}{|c|c|c|c|c|c|}
\hline \multirow[b]{2}{*}{ Commune } & \multirow[b]{2}{*}{$\begin{array}{c}\text { Nombre } \\
\text { de fermes testées }\end{array}$} & \multicolumn{3}{|c|}{ Résultat ELISA indirect } & \multirow[b]{2}{*}{$\begin{array}{c}\% \text { Fermes } \\
\text { séropositives }\end{array}$} \\
\hline & & $\begin{array}{c}\text { Positif } \\
\mathrm{PP} \geq 32 \%\end{array}$ & $\begin{array}{c}\text { Douteux } \\
\mathrm{PP}>26 \text { et }<32 \%\end{array}$ & $\begin{array}{c}\text { Négatif } \\
\mathrm{PP} \leq 26 \%\end{array}$ & \\
\hline Kimbaseke & 42 & 1 & 10 & 31 & 2,4 \\
\hline Kisenso & 28 & 11 & 5 & 12 & 39,3 \\
\hline Limete & 16 & 4 & 5 & 7 & 25 \\
\hline Masina & 17 & 0 & 3 & 14 & 0 \\
\hline Matete & 6 & 2 & 1 & 3 & 33,3 \\
\hline Mont-Ngafula & 155 & 80 & 28 & 47 & 51,6 \\
\hline Ndjili & 16 & 1 & 4 & 11 & 6,3 \\
\hline Ngaliema & 54 & 20 & 9 & 25 & 37 \\
\hline Nsele & 39 & 19 & 12 & 8 & 48,7 \\
\hline Selembao & 31 & 17 & 1 & 13 & 54,8 \\
\hline Total & 404 & 155 & 78 & & 38,4 \\
\hline
\end{tabular}

$\mathrm{PP}=$ pourcentage de positivité.

Tableau II. - Résultats des tests sérologiques réalisés dans les fermes de différentes communes. 


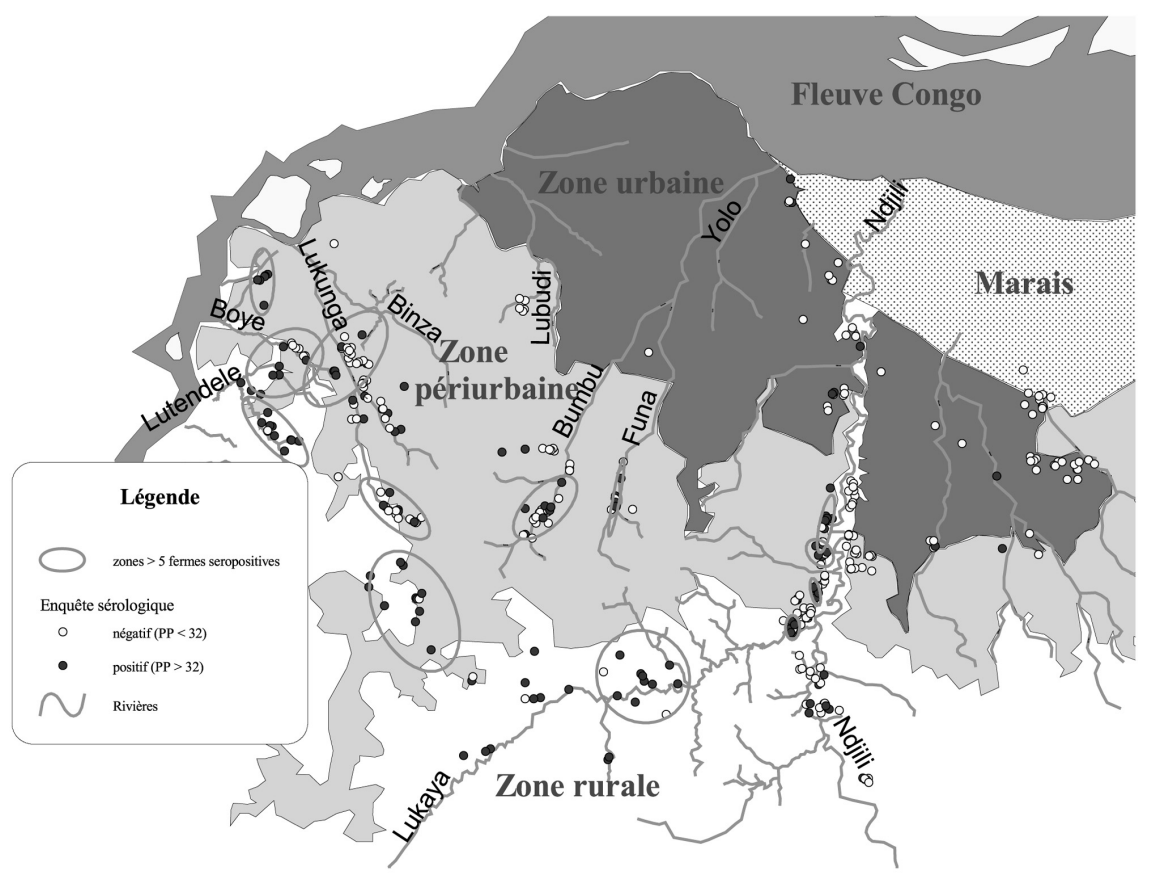

Fig. 2. - Distribution des élevages porcins séropositifs et les zones où ils sont agrégés.

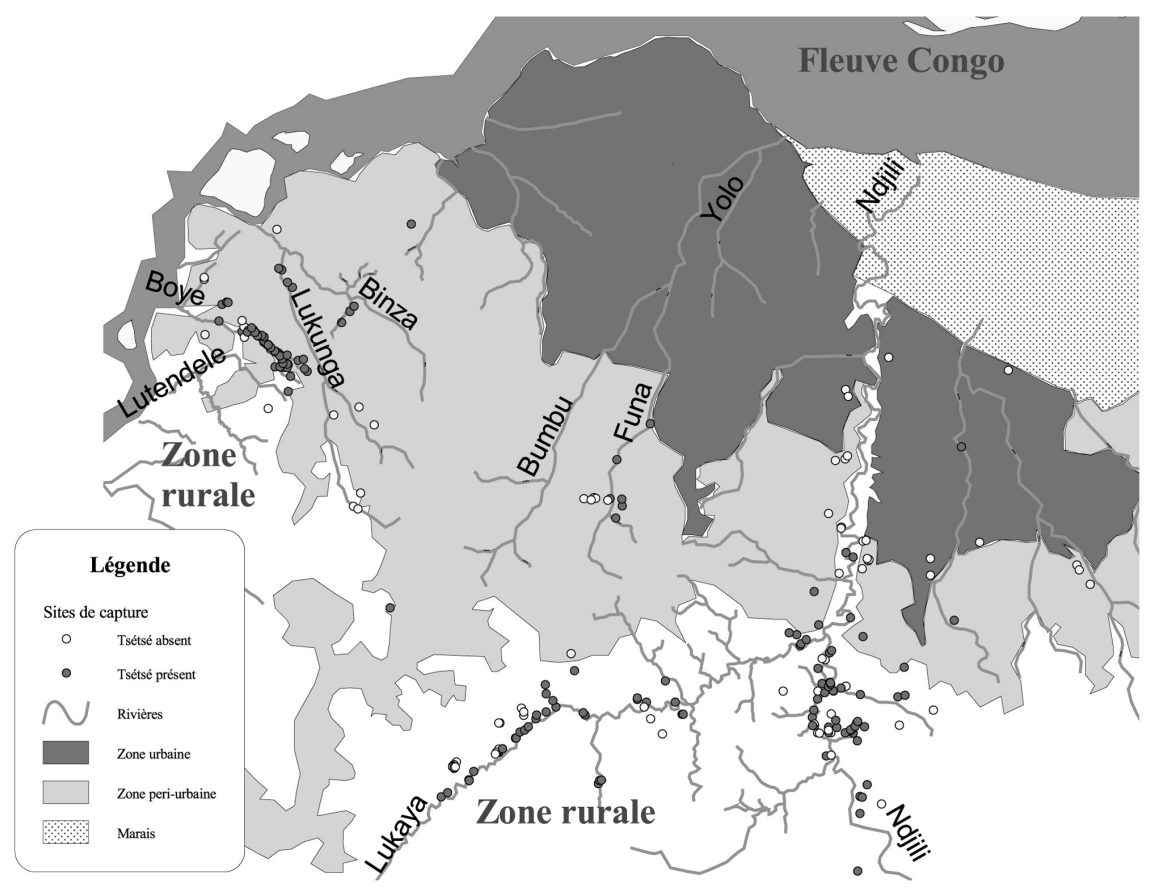

Fig. 3. - Sites où des mouches tsé-tsé ont été capturées près des élevages.

tification des espèces et sous-espèces de Trypanosoma sont en cours, mais d'ores et déjà il semble que Trypanosoma simiae joue un rôle prépondérant dans la trypanosomose porcine à Kinshasa.

\section{DISCUSSION}

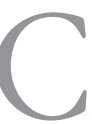

ette étude confirme que dans certains quartiers de Kinshasa la TAA est un réel problème pour l'élevage porcin. Au total, dans $38 \%$ des fermes prospectées, des animaux ont été trouvés positifs en
ELISA. Les fermes présentant des cas parasitologiquement confirmés ( $6 \%$ de toutes les fermes prospectées) se situent essentiellement dans la commune de MontNgafula. La corrélation de ces données avec la distribution des glossines permet la délimitation de quelques zones à haut risque. Le risque d'infection pour les porcs semble être élevé sur les bassins versants de certaines rivières (N’Djili, Lukaya, Boyé, Lutendele et Lukunga) dans la partie périurbaine de Kinshasa, où la présence des mouches tsétsé a été confirmée. Toutefois, aucune mouche tsé-tsé n'a été capturée le long de la rivière Bumbu, qui est située dans une zone d'habitation, où 
la galerie forestière a été détruite suite à l'urbanisation et aux activités de maraîchage et d'élevage.

Le taux de séropositivité de $45 \%$ chez le porc kinois est inférieur à celui de $56,3 \%$, publié par F. Noireau et al. (1986) et obtenu à partir du test CATT chez des porcs dans l'agglomération de Brazzaville touchée aussi par la maladie du sommeil et située sur l'autre rive du fleuve Congo.

Nos résultats entomologiques, avec 4,1\% des mouches infectées, rejoignent ceux de l'étude menée à Léopoldville (actuellement Kinshasa) par Van Hoof \& Henrard (1933). Le taux d'infection des glossines variait alors de 0,7 à plus de $4 \%$ selon la richesse du réservoir vertébré voisin. Leak \& Rowlands (1997) mentionnent également des taux d'infection similaires pour G.f. quanzensis qui s'élèvent de 0,1 à $5,4 \%$ pour $T$. vivax, de 0 à $0,2 \%$ pour $T$. congolense et de 0 à $0,01 \%$ pour $T$. brucei. Ces taux sont très bas comparés au taux d'infection de $28 \%$ rencontré chez Glossina p. palpalis (Robineau-Desvoidy, 1830) à Bonon en Côte d'Ivoire (Jamonneau et al., 2004). La plupart (92\%) des mouches infectées provenaient de la seule commune de Mont-Ngafula, qui était aussi le lieu où $12 \%$ des fermes présentaient des cas de TAA porcine parasitologiquement confirmés. Le taux élevé de mouches tsé-tsé à cet endroit pourrait s'expliquer par la densité du réseau hydrographique ainsi que la végétation faite essentiellement de galeries forestières jalonnant les cours d'eau dans des savanes parsemées d'arbustes, offrant un biotope favorable à la survie du vecteur. Le problème d'infestation par les tsé-tsé y était d'ailleurs tel qu'une organisation non gouvernementale locale avait déjà lancé une lutte antivectorielle dans ce secteur.

En comparant les résultats de capture des mouches tsétsé (figure 3) avec les résultats sérologiques (figure 2), il apparaît une correspondance entre la présence des glossines et les résultats sérologiques pour les communes de Mont-Ngafula, Ngaliema et N'Sele. Dans les communes de Mont-Ngafula et Ngaliema, dans la partie nord-ouest de Kinshasa au niveau de la zone périurbaine et sa transition avec la zone rurale, la présence de glossines a été confirmée le long des rivières Lukunga et Binza ainsi que sur les rivières Boyé et Lutendele. De même, au Sud de la zone d'étude, le long de la rivière Lukaya dans sa partie Nord, les glossines sont également relativement abondantes (De Deken et al, 2005). Cependant, la commune de Selembao reste une énigme car aucun vecteur n'y a été capturé, alors que les porcs qui y ont été prélevés montrent une prévalence élevée d'anticorps contre les trypanosomes. Toutefois, des investigations plus approfondies sur leur origine indiquent que la quasi-totalité des porcs charcutiers de cette zone ont vécus comme porcelets dans des communes infestées de tsé-tsé.

Au niveau de la rivière N'Djili, la majorité des fermes, où des animaux séropositifs ont été trouvés, se trouve le long de la rivière dans la zone périurbaine (N'Djili Cecomaf) et rurale (N'Djili Brasseries). Leur fréquence diminue avec la distance à la rivière. Ceci rejoint l'hypothèse selon laquelle Glossina fuscipes quanzensis ne s'éloigne peu de son biotope favorable.

Les corrélations spatiales entre séropositivité des porcs, infestation des mouches et présence des cas de TAA parasitologiquement confirmés, prouvent l'existence d'une transmission active de la TAA chez les porcs dans les communes de Mont-Ngafula (le long de la rivière Lutendele, Boyé et Lukaya) et de Ngaliema (le long de la rivière Lukunga).

La question persiste quant au risque pour l'homme de contracter la THA dans ces zones. Cette étude identifie Mont-Ngafula, comme une zone à risque accrue pour la TAA et c'est précisément dans cette zone, dans le quartier Lemba Imbu qu'avaient été dépistés en 1999, 160 cas de THA (De Deken et al, 2005). En outre, les enquêtes entomologiques effectuées par Simo (2006) ne confirment pas seulement les zones de Kinshasa, où les mouches sont présentes, mais démontrent par la biologie moléculaire la présence de Trypanosoma brucei gambiense dans 2,3\% des mouches capturées dans ces zones. Le Programme National de Lutte contre la Trypanosomose Humaine Africaine (PNLTHA) de la RDC a initié la lutte antivectorielle par piégeage des glossines pour réduire le contact homme-mouche à Kinshasa. Cependant, cette méthode rencontre encore des problèmes compte tenu du manque de personnel et des moyens insuffisants alloués à cette stratégie. Toutefois, nous avons noté que certains éleveurs, à petite échelle, utilisent aussi le piégeage comme moyen de lutte contre la TAA. Étant donné le caractère local du risque pour les porcs d'attraper la trypanosomose et les difficultés à traiter le porc atteint de T. simiae, une généralisation de la lutte antivectorielle semble une bonne stratégie dans ce contexte.

\section{REMERCIEMENTS}

T es auteurs présentent leur sincère gratitude à la Direction Générale de la Coopération au Développement (DGCD) de la Belgique pour la contribution financière de ce projet par l'entremise de l'Institut de Médecine Tropicale d'Anvers (IMTA)

\section{RÉFÉRENCES}

De Deken R., Sumbu J., Mpiana S., Mansinsa P., Wat'senga F., Lutumba P., Boelaert M. \& VAn Den Bossche. Trypanosomiasis in Kinshasa: distribution of the vector, Glossina fuscipes quanzensis, and risk of transmission in the peri-urban area. Medical and Veterinary Entomology, 2005, 19, 353359. 
Deckers N. Experimental infection of Trypanosoma congolense in pigs and a contribution to the improved diagnosis of trypanosomosis in pigs, MSc thesis Institute of Tropical Medicine of Antwerp, 2004.

Diouf S. Mise au point de la technique d'ELISA pour le diagnostic de la trichinellose et son utilisation par enquête épidémiologique. MSc thesis, Institute of Tropical Medicine of Antwerp, 2000.

Ebeja A.K., Lutumba P., Molisho D., Kegels G., Miaka mia Bilenge C. \& Boelaert M. La maladie du sommeil dans la région Ville de Kinshasa : une analyse rétrospective des données de surveillance sur la période 1996-2000. Tropical Medicine and International Health, 2003, 8, 945-955.

Greggio G. Trypanosomose des porcs : relation des porcs avec la trypanosomose humaine dans la vallée de l'Inkissi (Moyen Congo Belge). Bulletin de la Société de Pathologie Exotique et de ses Filiales, 1917, 10, 113-117.

Hirumi H. \& Hirumi K. In vitro cultivation of Trypanosoma congolense bloodstream forms in the absence of feeder cell layers. Parasitology, 1991, 102, 225-236.

Jamonneau V., Ravel S., Koffi M., Kaba D., Zeze D.G., Ndri L., Sane B., Coulibaly B., Cuny G. \& Solano P. Mixed infections of trypanosomes in tsetse and pigs and their epidemiological significance in a sleeping sickness focus of Cote d'Ivoire. Parasitology, 2004, 129, 693-702.

Kageruka P., Coelart J. \& Nkulu Pela N. Strain of Trypanosoma (Trypanozoon brucei) isolated from pigs in BasZaire. Annales de la Société Belge de Médecine Tropicale, 1977, 57, 85-88.

LEAK S.G.A. \& Rowlands G.J. The dynamics of trypanosome infections in natural populations of tsetse (Diptera: Glossinidae) studied using wing-fray and ovarian ageing techniques. Bulletin of Entomological Research, 1997, 87, 273-282.

LEVINE N. Crimestat II: a spatial statistics program for the analysis of crime incident locations. Ned Levine \& Associates, Houston, TX, and the National Institute of Justice, Washington, DC, 2002.

LuCKINS A.G. Detection of antibodies in trypanosome-infected cattle by means of a microplate enzyme-linked immunosorbent assays. Tropical Animal Health and Production, 1977, 9, 53-62.

LuCKINS AG. \& Mehlitz D. Evaluation of indirect fluorescent antibody test, enzyme linked immunosorbent assay and qualification of immunoglobins in the diagnosis of bovine trypanosomiasis. Tropical Animal Health and Production, 1978, 10, 149-159.

Makumyaviri A., Mehlitz D., Kageruka P., Kazyumba G.L. \& Molisho D. Le réservoir animal de Trypanosoma brucei gambiense au Zaïre : infections trypanosomiennes dans deux foyers du Bas-Zaïre. Tropical Medicine and Parasitology, 1989, 40, 259-261.

Murray M., MurRay P.K. \& McInTYRe W.I.M. An improved parasitological technique for the diagnosis of African trypanosomosis. Transactions of the Royal Society of Tropical Medicine and Hygiene, 1977, 71, 325-326.

NJIOKOu F. Wild fauna as a probable animal reservoir for Trypanosoma brucei gambiense in Cameroon. Infection Genetics and Evolution, 2006, 6, 147-153.

Nkinin S.W., Njiokou F., Penchenier L., Grebaut P., Simo G.
\& HeRDER S. Characterization of Trypanosoma brucei s. 1. subspecies by isoenzyme in domestic pigs from the Fontem sleeping sickness focus of Cameroon. Acta Tropica, 2002, 81, 225-232.

Noireau F., Gouteux J.P., Toudic A., Samba F. \& Frezil J.L. Importance épidémiologique du réservoir animal à Trypanosoma brucei au Congo : 1. Prévalence des trypanosomoses animales dans les foyers de maladie du sommeil. Tropical Medicine and Parasitology, 1986, 37, 393-398.

Rebeski D.E., Winger E.M., Lelenta M., Colling A., Robinson M.M., Ndamkou Ndamkou C., Aigner H., Dwinger R.H. \& CROWTHER J.R. Comparison of precoated and freshly coated microtitre plates using denaturated antigen for the detection of antibodies against Trypanosoma congolense by indirect enzyme-linked immunosorbent assay, in: Livestock production for rural development. Proceedings of the $\mathrm{IX}^{\text {th }}$ international conference of Association of Institutions of Tropical Veterinary Medicine, 14-18 September 1998, Harare, Zimbabwe. Volume I. Mukaratirwa S. \& Obwolo M.J. (eds), 1998, 376-386.

Robays J., Ebeja K., Lutumba P., Miaka mia Bilenge C., Kande Betu Ku Mesu V., De Deken R., Makabuza J., Deguerry M., VAN DER STUYFT \& Boelaert M. Human African trypanosomosis amongst urban residents in Kinshasa: a case-control study. Tropical Medicine \& International Health, 2005, 9, 869-874.

SChares G. \& Mehlitz D. Sleeping sickness in Zaire: a nested polymerase chain reaction improves the identification of Trypanosoma (Trypanozoon) brucei gambiense by specific kinetoplast DNA probes. Tropical Medicine E International Health, 1996, 1, 59-70.

Simo G., Asonganyi T., Nkinin S.W., Nuiokou F. \& Herder S. High prevalence of Trypanosoma brucei gambiense group 1 in pigs from the Fontem sleeping sickness focus in Cameroon. Veterinary Parasitology, 2006, 139, 57-66.

Simo G., Mansinsa P., Kande Betu Ku Mesu V., Manzambi E.Z., Ollivier G., Asonganyi T., Cuny G. \& Grébaut P. Human African trypanosomiasis transmission, Kinshasa, Democratic Republic of Congo. Emerging Infectious Diseases, 2006, 12, 1968-1970.

Steindl F., Armbruster C., Pierer K., Purtscher M. \& Kantinger H.W. A simple and robust method for the complete dissociation of HIV-p24 and other antigens from immune complexes in serum and plasma samples. Journal of Immunological Methods, 1998, 217, 143-151.

VAn HoOf L. \& HenRaRd C. Recherches sur les trypanosomes pathogènes du bétail à Léopoldville (Congo belge). Annales de la Société Belge de Médicine Tropicale, 1933, 13, 496-516.

WARBURG O. \& Christian W. Isolierung und Kristallisation des Gärungsferments Enolase. Biochem. Z., 1942, 310, 384-421.

Wright P.F., Nilsson E., VAn RoOij E.M.A., Lelenta M. \& JegGO M.H., Standardisation and validation of enzyme-linked immunosorbent assay techniques for the detection of antibody in infectious disease diagnosis. Revue Scientifique et Technique. Office Internationale des Épizooties, 1993, 12, 435-450.

Woo P.T.K. The haematocrit centrifuge technique for the diagnosis of African trypanosomiasis. Acta Tropica, 1970, 27, 385-386.

Reçu le 21 juillet 2008 Accepté le 6 avril 2009 